\title{
Construction of Multicopy Expression Vectors for Regulated Over- production of Proteins in Klebsiella pneumoniae and Other Enteric Bacteria
}

\author{
By DIETHELM KLEINER, † WYATT PAUL AND MIKE J. MERRICK* \\ AFRC-IPSR Nitrogen Fixation Laboratory, University of Sussex, Brighton BN1 9RQ, UK
}

(Received 20 January 1988)

\begin{abstract}
A number of expression vectors have been constructed to allow over-production of selected gene products in Klebsiella pneumoniae and other enteric bacteria. The plasmids use the strong hybrid trp-lac (tac) promoter for gene expression, which is regulated by the $l a c I^{\mathrm{Q}}$ allele of the lac repressor carried on the vector. This provides very tight regulation of gene expression, which is important for over-production of proteins which may be detrimental to cell growth. The vectors carry the standard mpl 8 cloning nest in which all the restriction sites are unique to the plasmid (with the exception of EcoRI in pDK7). Derivatives were constructed carrying kanamycin, chloramphenicol or ampillicin resistance as selectable markers, the first two of which are advantageous in $K$. pneumoniae due to the high inherent $\beta$-lactamase activity of this organism.
\end{abstract}

\section{INTRODUCTION}

In recent years a number of plasmid vectors have been developed for the purpose of overproducing particular gene products in Escherichia coli (Pouwels et al., 1985). These vectors have several potential applications, including the identification of gene products, studies on gene regulation, and synthesis of large amounts of a protein as an aid in its purification. In most situations the gene of interest is placed downstream of a strong promoter in order to generate high levels of the required transcript. Constitutive high-level expression is often undesirable as it can be detrimental to the host cell and frequently results in loss of the plasmid or selection of mutant plasmids which fail to express the product. Consequently many vectors have utilized either the lac promoter or variants of it (Amann et al., 1983) or the $\lambda \mathrm{p}_{\mathrm{L}}$ promoter (Remaut et al., 1981), both of which can be regulated. In the case of $\lambda p_{L}$ these vectors must be propagated in a host containing a thermolabile repressor protein (encoded by $\lambda c 1857$ ) and induction of gene expression is achieved by a temperature shift (usually $28^{\circ} \mathrm{C}$ to $42^{\circ} \mathrm{C}$ ) which inactivates the repressor. Expression from vectors carrying the lac promoter has been achieved by using host strains which overproduce the lac repressor, i.e. $l a c I^{\mathrm{Q}}$ mutants (Müller-Hill et al., 1968), and induction is achieved by addition of the lac inducer IPTG (Amann et al., 1983).

We have recently been interested in the application of these approaches to over-production of gene products in Klebsiella pneumoniae. As a result we have developed a number of new expression vectors which have certain advantages for work in this organism and potentially have wider application for over-production of proteins in other enteric bacteria. As at least one of the proteins we are interested in is temperature sensitive we have chosen to base our vectors on the hybrid tac promoter (Amann et al., 1983) and we have incorporated the $l a c I^{\mathrm{Q}}$ gene into the vectors so that their use is not restricted to specific $E$. coli strains. Furthermore, as nearly all previous expression vectors carry the bla $\left(\mathrm{Ap}^{\mathrm{r}}\right)$ gene as the selectable antibiotic resistance, and as $K$. pneumoniae is significantly more resistant to penicillin and its derivatives than $E$. coli, we

† Present address: Lehrstuhl für Mikrobiologie, Universität Bayreuth, 8580 Bayreuth, FRG.

Abbreviations: dNTP, deoxynucleoside triphosphate; X-gal, 5-bromo-4-chloro-3-indolyl $\beta$-D-galactoside; IPTG, isopropyl $\beta$-D-thiogalactopyranoside. 
have constructed alternative vectors carrying the cat $\left(\mathrm{Cm}^{\mathrm{r}}\right)$ or $k a n\left(\mathrm{Km}^{\mathrm{r}}\right)$ genes. In this paper we describe the construction of these vectors and their application for over-production of certain gene products in $K$. pneumoniae.

\section{METHODS}

Strains and plasmids. These are listed in Table 1.

DNA isolation and manipulation. Plasmid DNA was prepared by the method of Holmes \& Quigley (1981). Restriction endonucleases were obtained from commercial sources and used according to the manufacturer's instructions. Other DNA manipulations were carried out according to Maniatis et al. (1982). Transformation of plasmid DNA was carried out by the freeze/thaw procedure of Merrick et al. (1987).

Construction of expression vectors. Plasmid pMM40 was based on the ptac expression vector pKK223-3 (Pharmacia Ltd; Amann et al., 1983), which is derived from pBR322 and which carries the tac promoter followed by the $\mathrm{mp} 8$ cloning nest and a strong transcription terminator from the $E$. coli rrnB operon. In order to facilitate the use of this vector in $K$. pneumoniae, the $l a c I^{\mathrm{Q}}$ gene was isolated as an $A s p 718-P s t \mathrm{I}$ fragment from pMJR1560 (Stark, 1987) and cloned between the SphI and XmaIII sites of pKK223-3 (Fig. 1) after all the ends had been rendered blunt using Klenow polymerase and dNTPs. The ligation products were transformed into $E$. coli strain $5 \mathrm{~K}$, which is $\mathrm{Lac}^{+}$, and clones carrying $\mathrm{lacl}^{\mathrm{Q}}$ were selected as white colonies on plates containing the indicator $\mathrm{X}$-gal. The orientation of $l a c I^{\mathrm{Q}}$ was subsequently determined by restriction mapping.

Plasmid pMM63 was constructed in an analogous manner to pMM40 but in this case the lac $I^{\mathrm{O}}$ fragment was cloned between the SphI and PvuII sites of pKK223-3 (Fig. 2). pDK5 was derived from pMM63 in two steps.

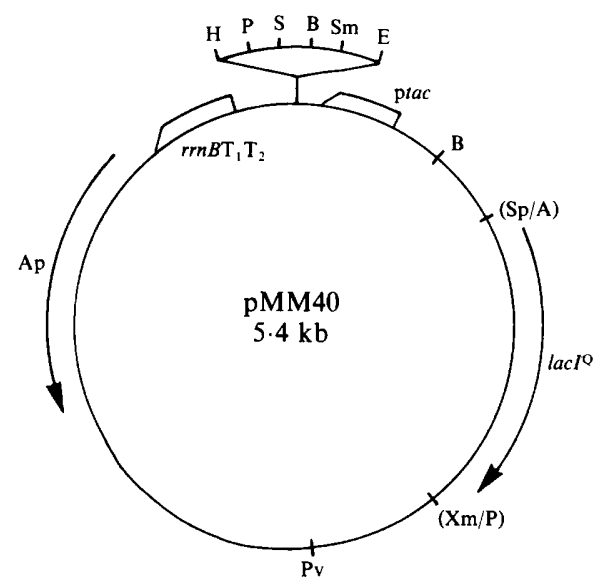

Fig. 1. Restriction map of pMM40. The lacI ${ }^{\mathrm{Q}}$ gene was cloned on an Asp718-PstI fragment into pKK223-3 cut with SphI and XmaIII; all ends were rendered blunt prior to ligation. Abbreviations for restriction sites in this and following Figures are: A, Asp718; Ah, AhaIII; B,BamHI; E, EcoRI; H, HindIII; Ha, HaeII; K, KpnI ; M, MluI ; P, PstI ; Pv, PvuII; S, SalI; Sa, SacI; Sm, SmaI; Sp, SphI; $\mathrm{X}, \mathrm{XbaI} ; \mathrm{Xm}, \mathrm{XmaIII}$. Hybrid sites are shown in parentheses.

Table 1. Bacterial strains and plasmids

Relevant characteristics

Source or reference

E. coli strains

$5 \mathrm{~K}$

MC1061

RB791

Plasmids

pKK223-3

pMJR1560

pBGS18

pACYC184 thi thr-1 leuB6 lacI ${ }^{+} Y 1$ tonA21 supE44 hsdR araD139 $\triangle($ ara, leu $) 7697 \triangle$ lacZ4 galU galK strA hsdR lacI ${ }^{\mathrm{Q}}$ lacL8 $h s d R$

ptac $r r n B \mathrm{~T}_{1} \mathrm{~T}_{2} \mathrm{Ap}^{\mathrm{r}}$; derivative of $\mathrm{pBR} 322$

lacI ${ }^{\mathrm{Q}} \mathrm{Ap}^{\mathrm{r}}$; derivative of $\mathrm{pUC18}$

$\mathrm{Km}^{\mathrm{r}}$ derivative of $\mathrm{pUC} 18$

$\mathrm{Km}^{\mathrm{r}} \mathrm{Cm}^{\mathrm{r}}$
B. Spratt, University of Sussex, UK

Casadaban \& Cohen (1980)

Amann et al. (1983)

Pharmacia Ltd

Stark (1987)

Spratt et al. (1986)

Chang \& Cohen (1978) 

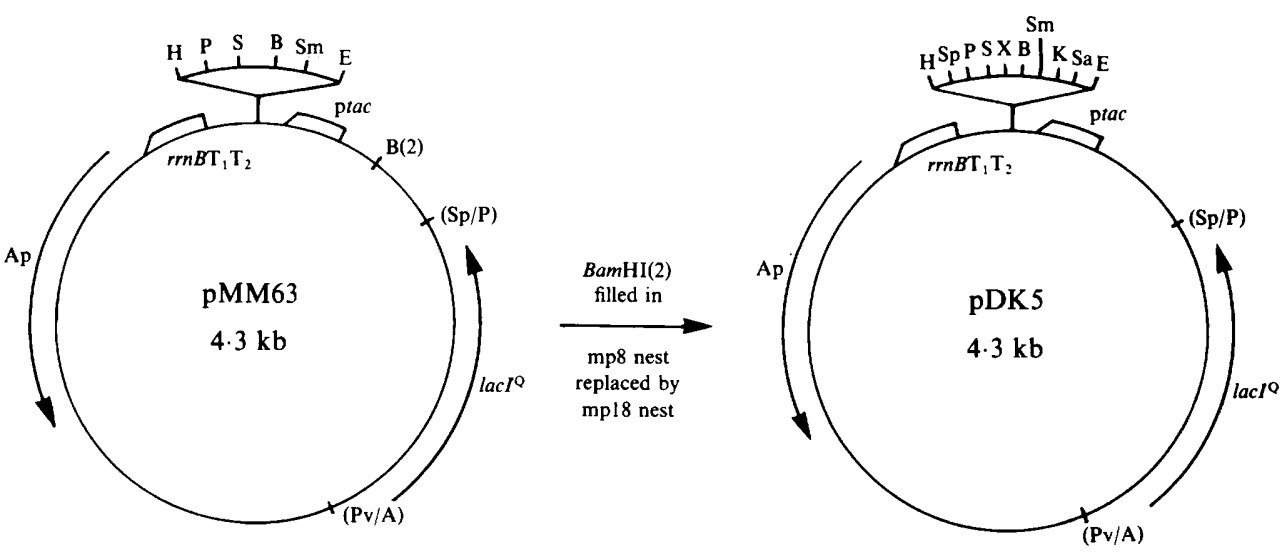

Fig. 2. Construction of pDK5. Plasmid pMM63 is analogous to pMM40 (Fig. 1) but lacI $I^{\mathrm{Q}}$ is inserted between $S p h \mathrm{I}$ and $P v u I I$ in the vector and is in the opposite orientation. pDK 5 was derived by filling-in $B a m H I(2)$ and replacing the cloning nest with that from pBGS18. Abbreviations for restriction sites are given in the legend to Fig. 1 .

(a)

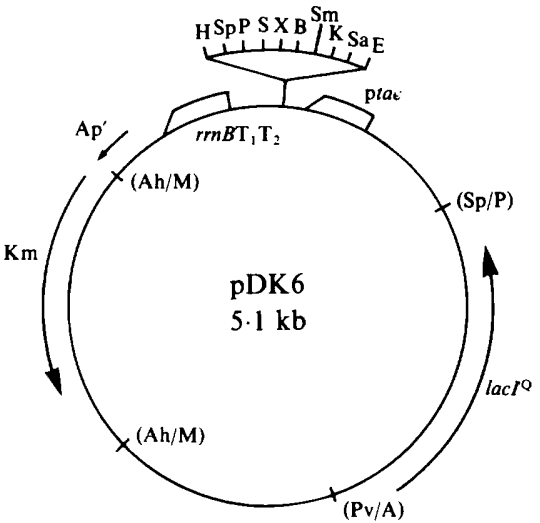

(b)

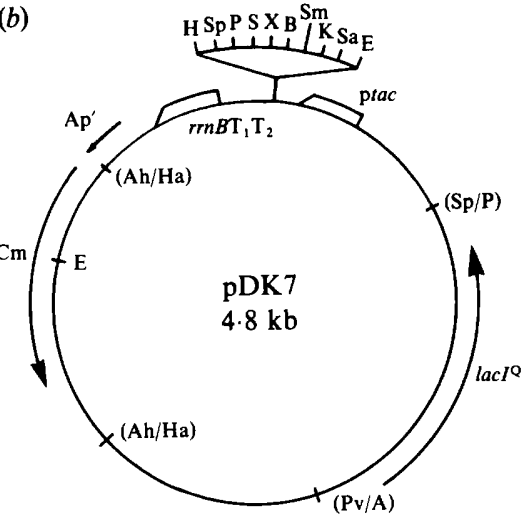

Fig. 3. Restriction map of (a) pDK6 and $(b)$ pDK7. The bla (Ap) gene of pDK5 was deleted using $A$ haIII and replaced with either the $k a n(\mathrm{Km})$ gene from $\mathrm{pBGS18}$ or the cat $(\mathrm{Cm})$ gene from pACYC184 to create pDK7. Abbreviations for restriction sites are given in the legend to Fig. 1.

Firstly the BamHI site outside the nest was removed by partial digestion with BamHI, filling in and religation followed by screening of individual clones to identify those in which the correct BamHI was removed. The cloning nest was then replaced by restricting with EcoRI and HindIII, and inserting the polylinker from pBGS18 (Spratt et al., 1986). The resultant plasmid pDK 5 is approximately $4.3 \mathrm{~kb}$ in size.

pDK6 and pDK 7 were derived from pDK 5 by restricting with AhallI to remove the majority of the bla gene, and cloning in either the 'filled-in' MluI fragment carrying the kan gene from pBGS18 to create pDK6, or the HaeII fragment (rendered blunt with Klenow polymerase) carrying the cat gene from pACYC184 to create pDK7 (Fig. 3). pDK6 and pDK 7 are $5 \cdot 1 \mathrm{~kb}$ and $4.8 \mathrm{~kb}$ in size respectively.

Induction of high-level gene expression. Cultures $(20 \mathrm{ml})$ of strains carrying the expression vectors were grown to exponential phase at $32{ }^{\circ} \mathrm{C}$ in Luria broth containing the appropriate selective antibiotic (carbenicillin $100 \mu \mathrm{g} \mathrm{ml}^{-1}$ for $E$. coli or carbenicillin $200 \mu \mathrm{g} \mathrm{ml}^{-1}+$ ampicillin $200 \mu \mathrm{g} \mathrm{ml}^{-1}$ for $K$. pneumoniae; kanamycin $15 \mu \mathrm{g} \mathrm{ml}^{-1}$ for $E$. coli or $30 \mu \mathrm{g} \mathrm{ml}^{-1}$ for $K$. pneumoniae; chloramphenicol $15 \mu \mathrm{g} \mathrm{ml}^{-1}$ for $E$. coli or $40 \mu \mathrm{g} \mathrm{ml}^{-1}$ for $K$. pneumoniae). For induction of ptac expression IPTG was added to a final concentration of $1 \mathrm{~mm}$ and incubation was continued at $32^{\circ} \mathrm{C}$. To analyse total cellular proteins $1 \mathrm{ml}$ samples were taken at $1 \mathrm{~h}$ intervals. Cells were pelleted and resuspended in $100 \mu \mathrm{l}$ Laemmli sample buffer, boiled for $5 \mathrm{~min}$ and analysed by SDS-PAGE using Coomassie brilliant blue $\mathrm{R}$ stain. 


\section{RESULTS}

Our initial experiments on protein over-production were concerned with the $n t r A$ gene of $K$. pneumoniae. This gene encodes an alternative RNA polymerase sigma factor $\left(\sigma^{54}\right)$ (Merrick \& Gibbins, 1985; Hunt \& Magasanik, 1985) and consequently over-production of this protein might be expected to be detrimental to cell growth. When $n t r A$ was cloned into pKK223-3 and the resultant plasmid was propagated in $E$. coli strain $\mathrm{RB} 791$, which carries a chromosomal $l a c I^{\mathrm{Q}}$ gene, over-production of $\sigma^{54}$ could be obtained after induction with IPTG but the plasmid was very unstable. Fast-growing sectors occurred at high frequency in colonies growing on Luria agar plates and mutant forms of the plasmid arose which could no longer be induced to overproduce. Subsequent experiments using multicopy plasmids carrying the lac $Z$ gene confirmed that a single chromosomal copy of $l a c I^{\mathrm{Q}}$ was insufficient to repress transcription completely from multiple copies of the lac promoter (data not shown). Consequently even in non-inducing conditions some over-production can occur and in the case of $\sigma^{54}$ this is sufficient to inhibit cell growth.

In order to overcome this problem and to allow regulated high-level expression in any strain of $K$. pneumoniae or $E$. coli the lacI $\mathrm{I}^{\mathrm{Q}}$ gene was cloned into pKK223-3 to generate pMM40 (Fig. 1). Plasmids carrying $l a c I^{\mathrm{Q}}$ inserted in either orientation were obtained and studies of the efficiency of ptac repression indicated that the level of repression was not affected by the orientation of lacI $I^{\mathrm{Q}}$ (data not shown). This type of plasmid was used to achieve regulated over-production of both the $n t r A$ product (on pMM38) and the $n t r B$ product (on pSA7) in response to IPTG induction in either $K$. pneumoniae or $E$. coli (Austin et al., 1987). After 2-3 h growth in the presence of IPTG the desired product constituted $1-2 \%$ of the total cell protein.

Plasmid pKK223-3 contains two BamHI sites, one in the cloning nest and one in the truncated tet gene still present in the plasmid (Fig. 1); both these sites are retained in pMM40. In order to improve pMM40 the BamHI site outside the nest was removed and the mp8 cloning nest was replaced by the mpl 8 nest (Fig. 2). In the resultant plasmid pDK 5, all the restriction sites in the cloning nest are unique. pDK 5 is also smaller than pMM40 because more of the pKK223-3 vector was deleted when $l a c I^{\mathrm{Q}}$ was inserted to produce pMM63, the predecessor of pDK5.

The complete DNA sequence of pDK 5 could be determined from its component parts and a detailed restriction analysis of the plasmid indicated that approximately $120 \mathrm{bp}$ were deleted at the Asp 718/PvuII junction during construction of pMM63. This deletion explains the absence of the Asp 718 site which was predicted to be retained at this junction. With this exception the restriction analysis of pDK 5 is entirely consistent with the expected sequence.

Although as mentioned previously pMM40 has been successfully used in $K$. pneumoniae for protein over-production, selection of ampicillin or carbenicillin resistance is difficult in many strains of Klebsiella owing to the high intrinsic level of $\beta$-lactamase produced by the host. Consequently alternative selectable markers are preferable for work in Klebsiella and for this reason derivatives of pDK 5 were constructed. Plasmids pDK6 and pKD7 carry the kan $\left(\mathrm{Km}^{\mathrm{r}}\right)$ and cat $\left(\mathrm{Cm}^{\mathrm{r}}\right)$ genes respectively cloned in the place of bla (Fig. 3) although the bla promoter and the first 212 bp of the bla coding sequence are retained in both plasmids. All the unique sites in the cloning nest are retained with the exception of EcoRI in pDK7, which is also present in the cat gene.

The utility of these pMM40 derivatives has been demonstrated by over-production of the $K$. pneumoniae nif $M$ product using plasmid pDK7. The nif $M$ gene has been sequenced recently (Paul \& Merrick, 1987) and shown to encode a $30.6 \mathrm{kDa}$ polypeptide. Both the bla gene and the $k a n$ gene encode polypeptides with molecular masses similar to that of NifM; $\beta$-lactamase (Bla) has a molecular mass of $31.5 \mathrm{kDa}$ and neomycin phosphotransferase (Kan) a molecular mass of $31.0 \mathrm{kDa}$. By comparision, chloramphenicol acetyltransferase (Cat) has a molecular mass of $25.7 \mathrm{kDa}$ and is therefore unlikely to confuse SDS-PAGE analysis of NifM synthesis. For these reasons nifM was cloned on a $0.9 \mathrm{~kb}$ Sst I-HpaI fragment between the $S s t \mathrm{I}$ and $S m a \mathrm{I}$ sites in the cloning nest of pDK7. The resultant plasmid, pWP12, was transformed into $E$. coli strain MC1061 and high-level expression of NifM was induced as described in Methods. After 1-3 h induction a polypeptide of the correct molecular mass for NifM was synthesized and constituted more than $1 \%$ of total cellular protein (Fig. 4). A second polypeptide was also produced at high 


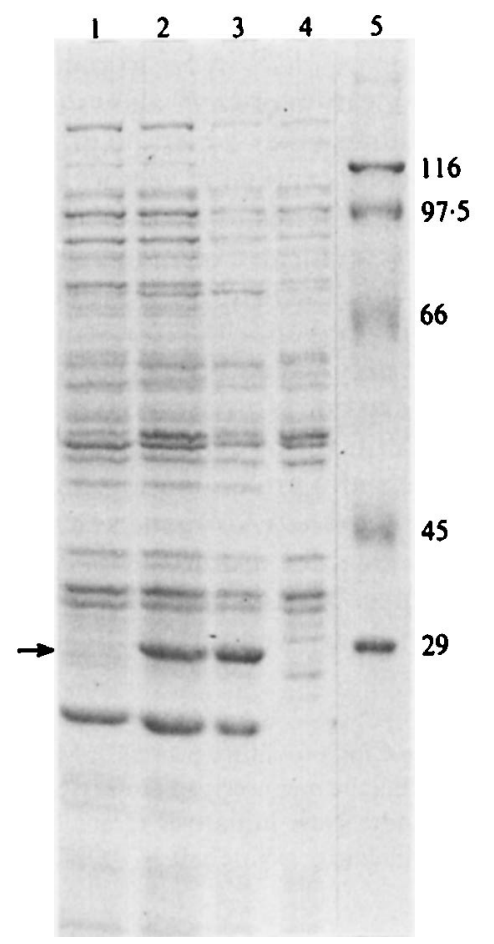

Fig. 4 Induction of NifM synthesis using plasmid pWP12 (based on pDK7) in E. coli strain MC1061. Cultures were grown and induced as described in Methods and total cell proteins were analysed by SDSPAGE. Track 1, prior to IPTG induction; tracks 2 and $3,1 \mathrm{~h}$ and $3 \mathrm{~h}$ respectively after IPTG induction; track 4, MC1061 alone $3 \mathrm{~h}$ after IPTG induction; track 5 , molecular mass standards. The NifM polypeptide is marked with an arrow.

levels in these strains even in the absence of induction. This polypeptide has the molecular mass expected of the cat product, and the high level of expression observed under all conditions in these experiments is probably due to the tandem arrangement of the bla and cat promoters in this plasmid.

\section{DISCUSSION}

The expression vectors described in this paper have a number of advantages for overproduction of specific gene products in enteric bacteria. The use of the tac promoter rather than the $\lambda \mathrm{p}_{\mathrm{L}}$ promoter to direct high levels of transcription provides a method of induction of gene expression (namely addition of IPTG) which is often preferable to the temperature shift required for $\lambda \mathrm{p}_{\mathrm{L}}$. Firstly, whilst a temperature shift is easily achieved on a small scale, i.e. cultures of 5-500 ml, it is far more difficult to obtain a comparable rapid shift when working on a large scale, e.g. 100-400 1. By comparison, addition of IPTG is simple and can effect efficient rapid induction. In large-scale cultures (e.g. $100 \mathrm{l}$ ) effective induction can be achieved with a final concentration of $100 \mu \mathrm{M}$-IPTG (S. Austin \& R. Dixon, personal communication). Secondly, if the activity of the protein being synthesized is temperature sensitive, induction with IPTG is clearly preferable to a temperature shift.

The location of the $l a c I^{\mathrm{Q}}$ gene on the expression vector has two distinct advantages. Firstly, as observed by ourselves and others (Amann et al., 1983; Stark, 1987) a single copy of lacI ${ }^{Q}$ is insufficient to repress expression from multiple copies of the tac promoter completely. Consequently without $l a c I^{\mathrm{Q}}$ on the plasmid there is always a significant level of expression from ptac and, as we found with the $\sigma^{54}$ product of $n \operatorname{tr} A$, this level of expression can be very 
detrimental to cell growth if the over-produced protein interferes with normal cellular metabolism. By contrast, the location of lacI $I^{\mathrm{Q}}$ in $c i s$ to $\mathrm{ptac}$ provides tight regulation of the inserted gene. Secondly the plasmid location of $l a c I^{\mathrm{Q}}$ allows the expression vector to be used in any chromosomal background including other genera, e.g. $K$. pneumoniae as described here.

The use of the $l a c I^{\mathrm{Q}}$ gene on multicopy expression vectors has also recently been described by Stark (1987), who constructed a similar series of plasmids based on the pUC vectors. These vectors (designated $\mathrm{pTTQ}$ ) have many features in common with the $\mathrm{pDK}$ vectors described here in that they utilize the $t a c$ promoter and the $r r B$ transcription terminator. The pTTQ vectors also contain the lac $Z \alpha$-complementing region which potentially allows the use of a blue-white colony colour screen (in the presence of X-gal and IPTG) to differentiate recombinant and nonrecombinant clones. However, this selection is often not possible since plasmids which express a desired gene at high levels may be difficult or even impossible to isolate under conditions of induction (Stark, 1987) and it is normally preferable to select transformants in non-inducing conditions. All the pTTQ vectors carry the bla $\left(\mathrm{Ap}^{r}\right)$ gene as a selectable marker and as discussed earlier this particular marker is not ideal for use in Klebsiella due to the intrinsic $\beta$-lactamase activity in this organism. In this respect pDK6 and pDK7 offer the advantage of using $\mathrm{Km}^{\mathrm{r}}$ or $\mathrm{Cm}^{\mathrm{r}}$ as selectable markers which may well be of benefit in other organisms besides K. pneumoniae.

The authors wish to thank Michael Stark for providing plasmid pMRJ1560 prior to publication. They also thank R. A. Dixon and B. E. Smith for reading the manuscript and Beryl Scutt for typing it. W. Paul acknowledges a research studentship from the AFRC under New Initiatives Grant NI 359/1 and D. Kleiner acknowledges financial support from the Deutsche Forschungsgemeinschaft.

\section{REFERENCES}

Amann, E., Brosius, J. \& Ptashne, M. (1983). Vectors bearing a hybrid trp-lac promoter useful for regulated expression of cloned genes in Escherichia coli. Gene 25, 167-178.

Austin, S., Henderson, N. \& Dixon, R. (1987). Requirements for transcriptional activation in vitro of the nitrogen-regulated $g \ln A$ and $n i f L A$ promoters from Klebsiella pneumoniae: dependence on activator concentration. Molecular Microbiology 1, 92-100.

Casadaban, M. \& Cohen, S. N. (1980). Analysis of gene control signals by DNA fusion and cloning in Escherichia coli. Journal of Molecular Biology 138, 179-207.

Chang, A. \& Cohen, S. N. (1978). Construction and characterisation of amplifiable multicopy DNA cloning vehicles derived from the p15A cryptic miniplasmid. Journal of Bacteriology 134, 1141-1 156.

Holmes, D. S. \& QuigleY, M. (1981). A rapid boiling method for the preparation of bacterial plasmids. Analytical Biochemistry 114, 193-197.

Hunt, T. \& MagasanIK, B. (1985). Transcription of glnA by purified Escherichia coli components: core RNA polymerase and the products of $g \ln F, g \ln G$ and glnL. Proceedings of the National Academy of Sciences of the United States of America 82, 8453-8457.

Maniatis, T., Fritsch, E. F. \& SambroOK, J. (1982). Molecular Cloning. A Laboratory Manual. Cold Spring Harbor, NY: Cold Spring Harbor Laboratory.

Merrick, M. J. \& Gibbins, J. R. (1985). The nucleotide sequence of the nitrogen-regulation gene ntrA of Klebsiella pneumoniae and comparison with conserved features in bacterial RNA polymerase sigma factors. Nucleic Acids Research 13, 7607-7620.

Merrick, M. J., Gibbins, J. R. \& Postgate, J. R. (1987). A rapid and efficient method for plasmid transformation of Klebsiella pneumoniae and Escherichia coli. Journal of General Microbiology 133, 20532057.

Müller-Hill, B., CRaPo, L. \& Gilbert, W. (1968). Mutants that make more lac repressor. Proceedings of the National Academy of Sciences of the United States of America 59, 1259-1264.

Paul, W. \& Merrick, M. (1987). The nucleotide sequence of the nif $M$ gene of Klebsiella pneumoniae and identification of a new nif gene - nifZ. European Journal of Biochemistry 170, 259-265.

Pouwels, P H., ENGer-ValK, B. E. \& Brammar, W. J. (1985). Cloning Vectors. A Laboratory Manual. Amsterdam: Elsevier.

Remaut, E., Stanssens, P. \& Fiers, W. (1981). Plasmid vectors for high-efficiency expression controlled by the $\mathrm{p}_{\mathrm{L}}$ promoter of coliphage lambda. Gene 15, 81-93.

Spratt, B. G., Hedge, P. J., Te Heesen, S., Edelman, A. \& BRoOME-SMith, J. K. (1986). Kanamycinresistant vectors that are analogues of plasmids pUC8, pUC9, pEMBL8 and pEMBL9. Gene 41, 337-342.

STARK, M. J. R. (1987). Multicopy expression vectors carrying the lac repressor gene for regulated highlevel expression of genes in Escherichia coli. Gene 51, 255-267. 